\title{
Influência da presença de metástase no perfil de mortalidade de pacientes oncológicos
}

\author{
Influence of the presence of metastasis on the mortality profile of cancer patients \\ Influencia de la presencia de metástasis en el perfil de mortalidad de pacientes \\ oncológicos
}

Luziane Fabiani ${ }^{1}$, Marciano Nascimento de Quadros $^{1}$, Malu Anton Eichelberger ${ }^{1}$, Angela Bocchese $^{2}$, Pablo Santiago ${ }^{1}$, José Afonso Corrêa da Silva ${ }^{1 *}$.

\section{RESUMO}

Objetivo: Identificar as principais causas de óbitos em pacientes oncológicos, conhecer os tumores mais incidentes, a influência das metástases no perfil e ainda a média de tempo de diagnóstico da doença até o óbito. Métodos: Foram analisados os prontuários de todos os pacientes oncológicos que foram a óbito no ano de 2014 em Hospital do Norte do Estado do Rio Grande do Sul. Resultados: A amostra foi composta de 244 pacientes, com predominância no sexo masculino $(n=133 ; 54,5 \%)$ e maioria dos óbitos com idade entre $70-79$ anos $(n=84 ; 34,4 \%)$ sem doença metastática, já na presença de metástase essa idade cai entre 50 - 59 anos com sobrevida de menos de 6 meses de vida. As neoplasias mais frequentes foram de pulmão, mama e estômago. A causa morte mais prevalente foi evolução da neoplasia ( $n=70 ; 30,3 \%$ ). Mais da metade dos pacientes estudados apresentavam alguma comorbidade associada, sendo que $32,4 \%$ $(n=79)$ detinham mais de duas comorbidades, dentre as mais encontradas cita-se a hipertensão arterial $(n=93)$, a diabete tipo $2(n=41)$ e cardiopatias $(n=17)$. Conclusão: Cada vez mais percebe-se a necessidade de prevenir o câncer e quando isso não for possível, que seja diagnosticado precocemente, evitando assim doenças mais avançadas e metastáticas, dando ao paciente sobrevida e qualidade de vida.

Palavras-Chave: Oncologia, Mortalidade, Metástase, Sobrevida.

\begin{abstract}
Objective: Identify the main death causes in cancer patients, to know the most occurring tumors, the prevalence of metastasis and if possible, the average time of the diagnosis of the disease until the time of death. Methodology: The medical records of all cancer patients who died in 2014 at Northern Hospital of the State of Rio Grande do Sul were analyzed. Results: The sample consisted of 244 patients, mosty male $(n=133 ; 54,5 \%)$ and most deaths around the age from 70 to 79 years aold $(n=84 ; 34,4 \%)$ without mestatic disease, in the presence of metastasis, this age drops to 50 to 59 years old with an extralife of less than 6 months. The most common cancers were lung, breast and stomach. The most prevalent cause of death was development of neoplasia $(n=70 ; 30.3 \%)$. More than half of patientis who were studied showed some related comorbidity, being that $32,4 \%(n=79)$ held more than two comorbidities, among the frequent are arterial hypertension $(n=93)$, diabete tipo $2(n=41)$ and heart diseases $(n=17)$. Conclusion: Now, more than ever, we realize the need to prevent cancer and if not possible, to diagnose as soon as possible, that way, avoiding more advanced and metastic diseases, giving the patient an extended life with quality.
\end{abstract}

Keywords: Oncology, Mortality, Metastasis, Survival.

\footnotetext{
${ }^{1}$ Hospital São Vicente de Paulo, Passo Fundo-RS. ${ }^{*}$ E-mail: joseafonso.hsvp@gmail.com

2 Universidade de Passo Fundo, Passo Fundo-RS.
} 


\section{RESUMEN}

Objetivo: Identificar las principales causas de muertes en pacientes oncológicos, conocer los tumores más incidentes, la influencia de las metástasis en el perfil y aún el promedio de tiempo de diagnóstico de la enfermedad hasta el óbito. En el presente estudio se analizaron los prontuarios de todos los pacientes oncológicos que fallecieron en el año 2014 en el Hospital del Norte del Estado de Rio Grande do Sul. Resultados: La muestra fue compuesta de 244 pacientes, con predominio en el sexo masculino ( $n=133$, El $54,5 \%)$ y la mayoría de las muertes con edad entre 70 y 79 años ( $n=84,34,4 \%$ ) sin enfermedad metastásica, ya en presencia de metástasis esa edad cae entre 50 - 59 años con sobrevida de menos de 6 meses de vida. Las neoplasias más frecuentes fueron de pulmón, mama y estómago. La causa muerte más prevalente fue la evolución de la neoplasia $(n=70 ; 30,3 \%)$. La mayoría de los pacientes estudiados presentaban alguna comorbilidad asociada, siendo que $32,4 \%(n=79)$ tenían más de dos comorbilidades, entre las más encontradas se cita la hipertensión arterial $(n=93)$, la diabetes tipo $2(n=93)=41)$ y las cardiopatías $(n=17)$. Conclusión: Cada vez más se percibe la necesidad de prevenir el cáncer y cuando eso no sea posible, que sea diagnosticado precozmente, evitando así enfermedades más avanzadas y metastásicas, dando al paciente sobrevida y calidad de vida.

Palabras-clave: Oncología, Mortalidad, Metástasis, Sobrevida.

\section{INTRODUÇÃO}

O câncer é um dos piores problemas de saúde pública e a causa mais grave da morte nos países desenvolvidos e em desenvolvimento, sendo responsável por mais de seis milhões de óbitos a cada ano, representando cerca de $12 \%$ de todas as causas de morte no mundo. Entretanto, a taxa geral de mortalidade por câncer aumentou durante a maior parte do século 20, em grande parte impulsionada pelo rápido aumento nas mortes por câncer de pulmão entre os homens como consequência da epidemia de tabaco, mas diminuiu cerca de 1,5\% ao ano desde a década de 1990 (SIEGEL RL et al., 2017).

Com o salto quantitativo demográfico estima-se que, até o ano de 2020, mais de dez milhões de pessoas no mundo tenham neoplasia, superando assim, as doenças cardiovasculares, tornando-se a maior causa de morte no mundo. Em aproximadamente 20 anos, as tendências apontam para um crescimento ainda mais expressivo deste índice, cerca de 100\%, com mais de 70\% dos casos novos e mortes ocorrendo nos países economicamente menos desenvolvidos, principalmente nas regiões onde há precárias condições de acesso aos serviços de saúde, principalmente voltados a identificação de fatores de risco (DATASUS, 2018; INCA, 2018).

Dentre esses fatores, o tabagismo, como um dos principais, não contribui somente para o aumento da carga de câncer de pulmão (BARROS JA, et al., 2006), mas também para o aumento da incidência de outros tipos de câncer, tais como de laringe, esôfago, boca e faringe, os dois últimos, principalmente se associado a consumo de álcool e precárias condições de nutrição, outros fatores de risco muito comuns no Brasil (MENEZES AM et al., 2002; TAZIKI MH et al., 2018).

Sem dúvida nenhuma, um dos pontos mais importantes, quando o objetivo é um prognóstico favorável no câncer, é o diagnóstico precoce, a fim de evitar o processo de metástase (FERREIRA AC et al., 2002), quando as células cancerosas de um tumor se espalham para diferentes partes do corpo, formando tumores satélites, distantes do tumor original. Este quadro por si só, já representa um agravante para a piora do quadro clínico (INCA, 2018) e para a utilização de drogas mais avançadas contra o tumor (JINDAL V, et al., 2018; PASQUALI S et al., 2018), aumentando consideravelmente a mortalidade associada a doença (INCA, 2018).

Estudos anteriores demonstraram que a taxa de mortalidade em Unidade de Terapia Intensiva (UTI) é significativamente maior entre pacientes oncológicos que os não-oncológicos em mesmas condições 
(PARKIN DM et al., 2001). Associado a essa condição, a presença de metástase apresenta indício relevantes, de que é determinante na mortalidade dos pacientes (FRANCESHCINI JP et al., 2017).

Sendo assim, este estudo tem por objetivo avaliar a influência da presença de metástase no perfil de mortalidade de pacientes oncológicos, assim como verificar quais as principais causas de óbitos, tumores mais incidentes e tempo de sobrevida dos pacientes.

\section{MÉTODOS}

Trata-se de um estudo transversal, exploratório e descritivo, a partir de prontuários eletrônicos de pacientes oncológicos que evoluíram ao óbito no período de janeiro a dezembro de 2017, em Hospital do Norte do Estado do Rio Grande do Sul, tendo como objetivo avaliar a influência da presença de metástase na probabilidade de sobrevivência do paciente, utilizando como parâmetro, o tempo entre o diagnóstico e o óbito.

Foram incluídos na pesquisa pacientes em tratamento oncológico, internados no Hospital em que foi realizada a pesquisa, no ano de 2017 e que evoluíram para óbito, excluindo então, pacientes sem doença oncológica ou que ainda estejam em tratamento oncológico.

O estudo foi realizado com o preenchimento de uma ficha pré-elaborada pelo pesquisador, no qual foram coletados os dados referentes ao gênero, faixa etária, causa do óbito, número e tipo de comorbidades, presença de metástase, tempo de sobrevivência e tipo da neoplasia. Todos os dados foram coletados direto do sistema informatizado do hospital.

Para elaboração do banco de dados e análise descritiva, foi utilizado o software SPSS 20.2 for Windows e para os testes de significância foi utilizado o teste não paramétrico Kruskall-Wills, considerando p=0,05.

O projeto foi aprovado pela Comissão Científica de Pós-graduação (CPPG) do hospital e pelo Comitê de Ética em pesquisas da Universidade de Passo Fundo (CEP-UPF) número 1.162.492. Não necessitando de "Termo de consentimento livre esclarecido".

\section{RESULTADOS}

A amostra foi composta de 244 pacientes que preencheram os critérios de inclusão (Tabela 1). A idade predominante dos pacientes foi entre 70-79 anos com 34,4\% $(n=84)$. A distribuição dos pacientes entre os sexos foi predominante no sexo masculino com $54,5 \%(n=133)$. As causas de morte mais prevalentes na amostra foram à própria evolução da neoplasia $(30,3 \%, n=74)$, falência múltipla dos órgãos e insuficiência respiratória, com $24,2 \%(n=59)$ e $23,0 \%(n=56)$, respectivamente. Mais da metade dos pacientes estudados apresentavam alguma comorbidade associada, sendo que $32,4 \% \quad(n=79)$ detinham de mais de duas comorbidades, dentre as mais encontradas observa-se a hipertensão arterial, a diabetes tipo 2 e cardiopatias.

Dentre os diagnósticos, o mais comum foi o de neoplasia de pulmão com 15,2\% ( $n=37$ ), seguida da neoplasia gástrica com $9,0 \%(n=22)$ do total de pacientes. O restante consiste nas neoplasias de pâncreas $10,7 \%(n=26)$, mama $9,4 \%(n=23)$ e próstata $6,6 \%(n=16)$. Um achado importante foi a presença de metástase em $53,7 \%(n=131)$ dos pacientes, sendo que as mais predominantes foram as metástases ósseas com 15,2\% ( $n=37)$, hepáticas $14,3 \%(n=35)$ e pulmonares com $11,1 \%(n=27)$.

A faixa etária entre 50-59 anos (Figura 1) apresentou um aumento significativo ( $p=0,049)$ de pacientes com menos de seis meses de sobrevida com a presença de metástase, em comparação aos pacientes sem presença de metástase na mesma faixa etária. Porém, foi na faixa etária de 70-79 anos em que um maior número de pacientes apresentou tempo de sobrevida menor que seis meses. 
Tabela 1 - Dados sociodemográficos de pacientes oncológicos que evoluíram ao óbito no período de janeiro de 2014 a dezembro de 2014 ( $n=244)$.

\begin{tabular}{lc}
\hline Características da amostra & $\mathbf{N}(\%)$ \\
\hline Sexo & $111(45,5)$ \\
\hline Feminino & $133(54,5)$ \\
\hline Masculino & \\
\hline Faixa etária & $5(2,0)$ \\
\hline$<20$ anos & $7(2,9)$ \\
\hline $20-29$ anos & $5(2,0)$ \\
\hline $30-39$ anos & $16(6,6)$ \\
\hline $40-49$ anos & $46(18,9)$ \\
\hline $50-59$ anos & $56(23,0)$ \\
\hline $60-69$ anos & $84(34,4)$ \\
\hline $70-79$ anos & $25(10,2)$ \\
\hline$>80$ anos &
\end{tabular}

Causa da morte

\begin{tabular}{lc}
\hline Falência múltipla dos órgãos & $59(24,2)$ \\
\hline Insuficiência respiratória & $56(23,0)$ \\
\hline Choque séptico & $37(15,2)$ \\
\hline $\begin{array}{l}\text { PCR (Parada } \\
\text { Cardiorrespiratória) }\end{array}$ & $18(7,4)$ \\
\hline Evolução da neoplasia & $74(30,3)$
\end{tabular}

\section{Número de comorbidades} associadas

\begin{tabular}{lc}
\hline Nenhuma comorbidade & $106(43,4)$ \\
\hline Uma comorbidade apenas & $59(24,2)$ \\
\hline Duas comorbidades apenas & $48(19,7)$ \\
\hline Três comorbidades apenas & $29(11,9)$ \\
\hline Quatro comorbidade apenas & $2(0,8)$ \\
\hline
\end{tabular}

Fonte: Dados da pesquisa, 2018.

Como mostra a Figura 2, apesar de diferença não significativa, os pacientes sem metástase apresentaram uma curva de sobrevida acima que a dos pacientes com metástase, demonstrando que em um mesmo período de tempo, o primeiro grupo, aparentemente, tem maior probabilidade de sobrevivência. 
Figura 1 - Influência da presença de metástase no tempo de sobrevida de pacientes oncológicos desde o diagnóstico.

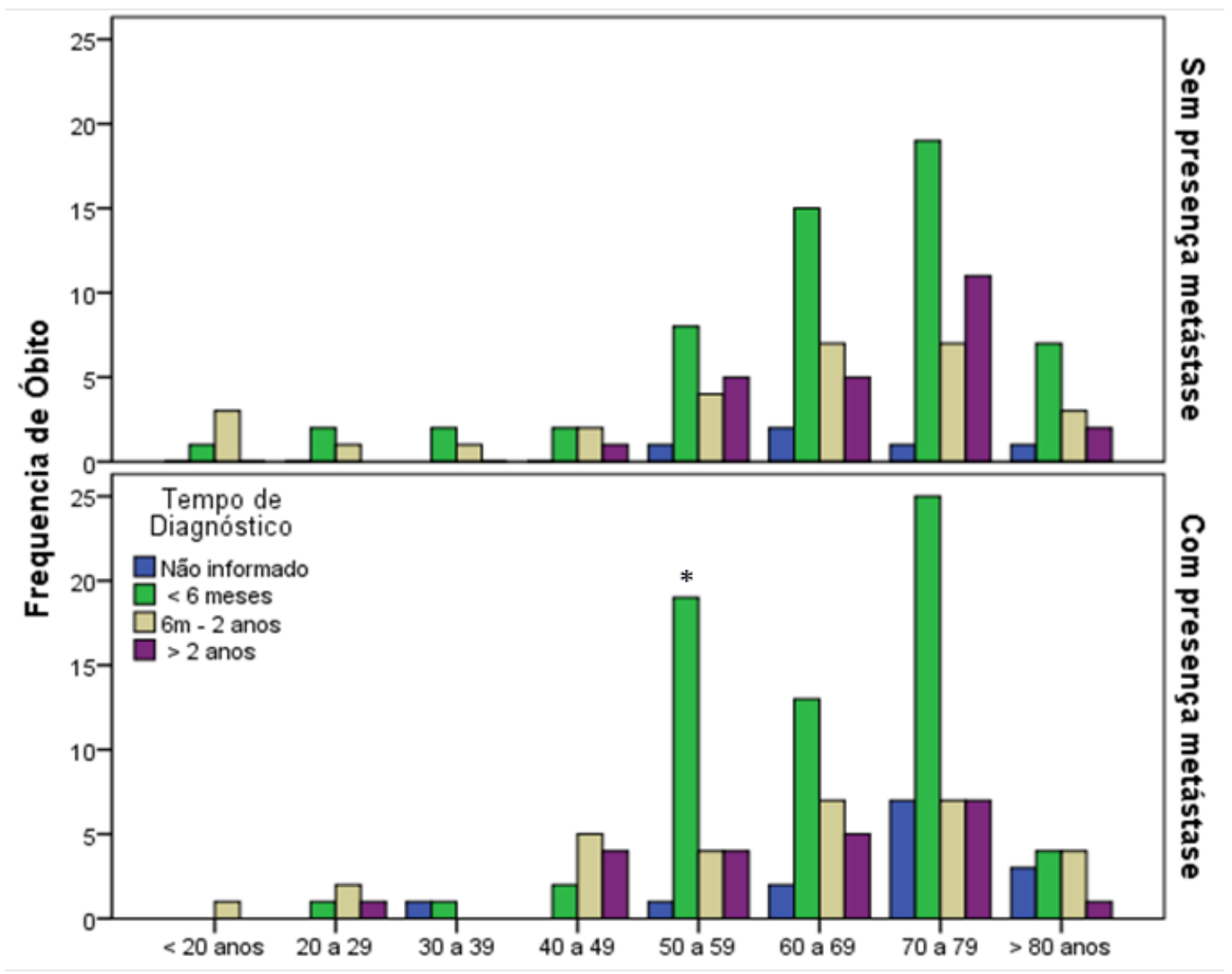

Fonte: Dados da pesquisa, 2018.

${ }^{*} p<0.05$, comparando o tempo de diagnóstico de pacientes da faixa etária de 50-59 anos com ou sem presença de metástase.

Figura 2 - Probabilidade de sobrevida de pacientes com/sem metástase.

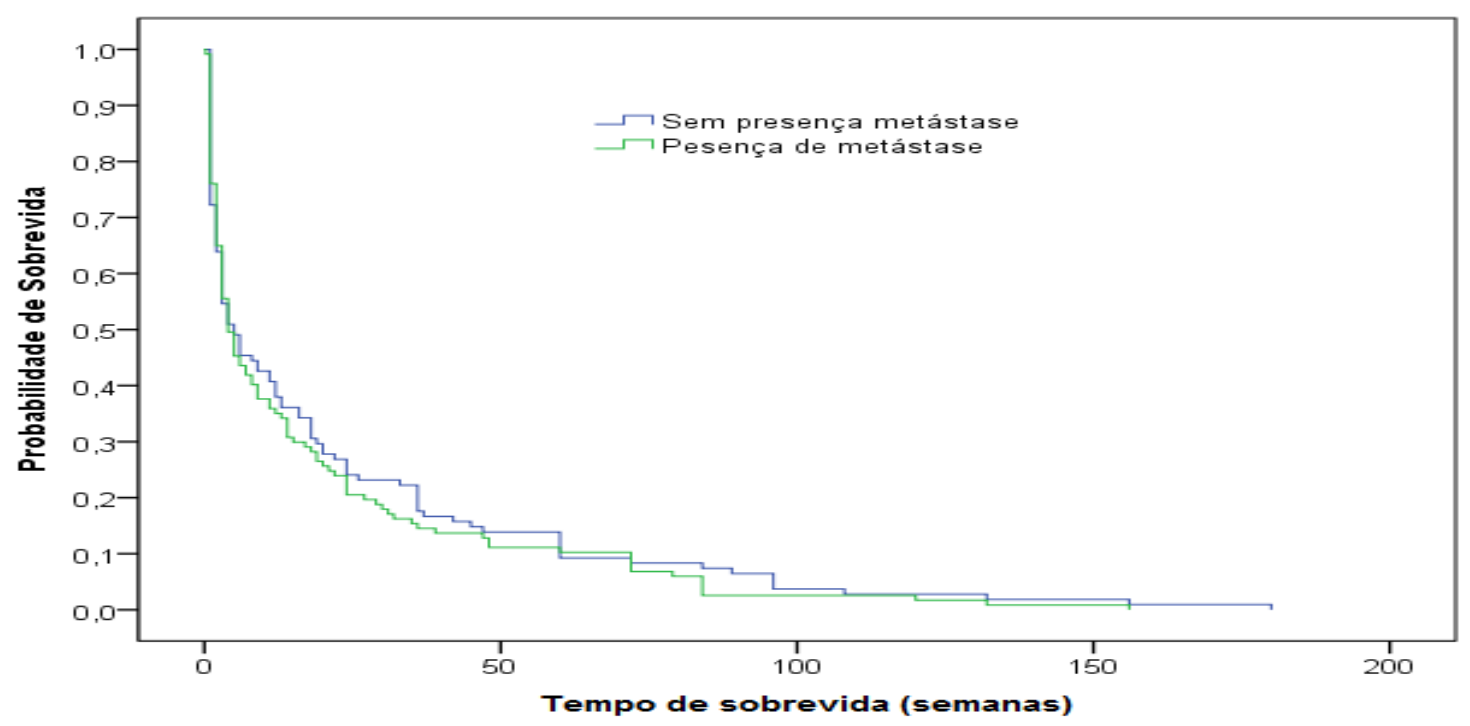

Fonte: Dados da pesquisa, 2018. 


\section{DISCUSSÃO}

A presença de metástase na condição clínica oncológica, sinaliza que há uma implantação de um foco tumoral à distância do tumor original, decorrente da disseminação do câncer para outros órgãos. Esta alteração celular, ocorre quando as células cancerígenas se desprendem do tumor primário e entram na corrente sanguínea ou no sistema linfático, podendo circular pelo organismo e se estabelecer em outro órgão (TALMADGE JE e FIDLER IJ 2014; MICALIZZI DS et al., 2017). Este estágio da doença é considerado grave e eleva a chance do surgimento de novas comorbidades associadas ao tumor primário, assim como aumenta a probabilidade de óbito pela doença (INCA 2018).

Existem diversos sítios passíveis de invasão por células cancerígenas, como por exemplo o tecido ósseo, acometido por metástases de tumores sólidos, incluindo pulmão, mama, próstata, colorretal e tireoide (FORNETTI J et al., 2018), o tecido hepático (JONES RP et al., 2013) e o parênquima pulmonar, este, originado de uma disseminação hematogênica, linfática, do espaço pleural, pelas vias aéreas ou por invasão direta (HIRAKATA K et al., 1995). A capacidade de disseminação da doença no organismo, resultando em um mal prognóstico e a pandemia mundial dos novos casos de câncer, fazem com que seja uma das principais causas de morte no mundo (DATASUS 2018; INCA 2018).

Com relação aos dados obtidos na pesquisa, houve uma predominância do gênero masculino, em contraste a outros estudos em países desenvolvidos, que demonstram um incidência de câncer considerada similar em ambos os gêneros, ou estudo que demonstraram o contrário, como no Brasil, onde há uma prevalência no sexo feminino de até $25 \%$ maior (PISANI P, et al., 2002).

Das neoplasias mais presentes, houve um predomínio da neoplasia de pulmão (15,2\%; n=37), neoplasia de mama $(11,5 \% ; n=28)$ e neoplasia gástrica $(9,0 \% ; n=22)$, apesar da maioria dos estudos demonstrarem que há um declínio do número de câncer de estômago nos últimos anos, utilizando como fundamento, hipóteses relacionadas à introdução da refrigeração; às mudanças nas técnicas de preservação de alimentos (usando menos sal e alimentos menos condimentados); ao consumo de frutas e de vegetais frescos; e à diminuição da prevalência da infecção por Helicobacter pylori. (LATORRE MR, 1997). Essa queda de mortalidade do câncer de estômago, indiferente do gênero, é constatado em numerosos países, incluindo alguns latino-americanos (FONSECA LAM et al., 2010).

Na região Sul, segundo as estimativas de 2014/2015 o câncer gástrico foi o quarto mais frequente em homens $(16,07 / 100 \mathrm{mil})$ e o sexto em mulheres $(8,43 / 100 \mathrm{mil})$. As demais neoplasias predominantemente encontradas no estudo, concordam com dados do Instituto Nacional de Câncer, que mostram a prevalência do câncer de pulmão com risco estimado de 16,79 casos novos a cada 100 mil homens e 10,75 a cada 100 mil mulheres e mama são esperados 57.120 casos novos de câncer de mama, com um risco estimado de 56,09 casos a cada 100 mil mulheres (INCA 2018).

As metástases mais encontradas foram às ósseas (15,2\%; $n=37)$, hepáticas $(14,3 \% ; n=35)$ e pulmonares $(11,1 \% ; n=27)$. A presença de metástase óssea pode ser explicada pela elevada incidência de câncer de mama na amostra, o maior causador desse tipo de metástase (FORNETTI J et al., 2018), assim como os sarcomas causadores de metástases pulmonares (DONATO J 2001).

Ainda assim, o tecido hepático, devido a sua alta vascularização e função metabólica, é o órgãos mais acometido a distância ( $40 \%$ a $50 \%$ ) e o que mais determina a sobrevida do paciente (NETO OCLF 2014).

Dos pacientes tratados com intenção curativa, $50 \%$ a $60 \%$ apresentaram recidiva local, regional ou à distância, demonstrando, como já citado na literatura, a dificuldade em impedir o progresso da doença e com isso, muitas vezes, o surgimento de metástase (TALMADGE JE e FIDLER IJ, 2014). Mukherjee S (2010), conclui que a única maneira de lidar com este problema, usando as abordagens atuais, é garantindo que o tratamento permaneça um passo à frente do surgimento de metástases, o que é muitas vezes intangível, já que em muitos pacientes, no momento do diagnóstico, a metástase já se faz presente (TALMADGE e FIDLER, 2014). 
O menor tempo de sobrevida encontrado foi na faixa etária de 70-79 anos, não havendo diferença significativa quando comparado pacientes com ou sem a presença de metástase. Na faixa etária de 50-59 anos a presença de metástase influenciou significativamente o tempo de sobrevida dos pacientes. Uma explicação para esse fato é de que os pacientes na faixa etária acima de 70 anos sofrem maior influência das comorbidades associadas e das disfunções orgânicas, do que da presença ou não de metástase, como fator limitante da sobrevida, visto que nesta fase se torna mais comum a progressão da doença e das intercorrências associadas, como a insuficiência respiratória, que por exemplo, em uma Unidade de Terapia Intensiva (UTI), é responsável por $75 \%$ da mortalidade de pacientes oncológicos, comprovado pelo presente estudo onde correspondeu a $23 \%$ dos óbitos (PINHEIRO CTS e BRITTO L, 2001).

Como demonstrado na figura 2, em um mesmo período de tempo, o primeiro grupo (pacientes sem metástases) teve maior probabilidade de sobrevivência. Em estudo realizado com pacientes de câncer de cabeça e pescoço, os dados são compatíveis, mostrando menor sobrevida dos pacientes com metástases à distância e após certo tempo de tratamento, os pacientes com metástases e sem metástases se igualam, não fazendo diferença a presença ou não para o óbito (MANFRO $G$ et al., 2006).

Os resultados demonstram que os óbitos por doença oncológica tiveram predomínio no sexo masculino, com $54,5 \%(n=133)$ e ainda a presença de metástase como grande influência na sobrevida dos pacientes, fazendo com que a idade dos óbitos caia da faixa etária predominante da amostra de 70-79 anos para 5059 anos, e com sobrevida de menos de 6 meses.

Estudos desse tipo podem contribuir na implementação de políticas públicas, visando a programação de medidas nos diferentes níveis de atenção à saúde e melhoria da assistência a população assistida pelo SUS nos municípios e hospitais, além de colaborar com a escassa literatura sobre o perfil epidemiológico das neoplasias no atendimento público.

\section{CONCLUSÃO}

De fato, a presença de metástase na doença oncológica, é um fator limitante para um bom prognóstico e evolução do tratamento. Além de trazer complicações associadas ao tumor primário. Os achados da pesquisa evidenciam a necessidade de avançar com novos estudos de correlação, entre a presença de metástase e a mortalidade, visto que neste estudo, o grupo de pacientes de 50-59 anos com metástase, apresentou uma diminuição significativa do tempo de sobrevida e que de maneira não significativa, porém destacada, os pacientes com metástase obtiveram uma linha de sobrevida abaixo dos pacientes sem metástase. Baseando-se nos estudos celulares do quadro de metástase e nos dados epidemiológicos da doença, é sábio dizer, que todos os esforços têm que ser concentrados na prevenção do surgimento de metástase, e até, na diminuição dos fatores de risco para o surgimento da neoplasia.

\section{REFERÊNCIAS}

1. BARROS JA, et al. Diagnóstico precoce do câncer de pulmão: o grande desafio. Variáveis epidemiológicas e clinicas, estadiamento e tratamento. J Bras Pneumol, 2006;32(3):22127.

2. DATASUS. Banco de dados do Sistema Único de Saúde, 2009 (acesso em 20 set 2018). Disponível em: http://datasus.gov.br.

3. DONATO J. Tumores ósseos. In: Metástase de carcinoma. Rio de Janeiro: Rocca, 2001,p.211-6.

4. FERREIRA AC, et al. Contribuição da ultra-sonografia abdominal no seguimento de pacientes pós tratamento de câncer mamário. Radiol Bras, 2002;35(6):345-9.

5. FONSECA LAM, et al. Tendências da Mortalidade por Câncer nas Capitais dos Estados do Brasil, 1980-2004. Rev Assoc Med Bras, 2010; 56(3):309-12.

6. FORNETTI J, et al. Understanding the Bone in Cancer Metastasis. J Bone Miner Res, 2018;33(12):2099-13. 
7. FRANCESCHINI JP, et al. Sobrevida em uma coorte de pacientes com câncer de pulmão: papel da idade e do sexo no prognóstico. J Bras Pneumol, 2017;43(6):431-436.

8. HIRAKATA K, et al. CT of pulmonary metastases with pathological correlation. Semin Ultrasound CT MR, 1995;16:379-94.

9. INCA (Instituto Nacional do Câncer). Ministério da Saúde. Estimativas 2018: incidência do Câncer no Brasil, 2019 (acesso em 20 set 2018). Disponível em: http://www.inca.gov.br/estimativa/2018/casos-taxas-brasil.asp.

10. JINDAL V, GUPTA S. Expected Paradigm Shift in Brain Metastases Therapy-Immune Checkpoint Inhibitors. Mol Neurobiol, 2018;55(8):7072-78.

11. JONES RP, et al. Perioperative chemotherapy for resectable colorectal liver metastases: Where now? Eur J Surg Oncol. 2013;39(8):807-11.

12. LATORRE MR. Stomach cancer mortality in Brazil: from 1977 to 1989. Cad Saude Publica, 1997;13(1):12-16.

13. MANFRO G, et al. Relação entre idade, sexo, tratamento realizado e estágio da doença com a sobrevida em pacientes terminais com carcinoma epidermóide de laringe. Rev Bras Can 2006; 52(1): 17-24.

14. MENEZES AM, et al. Risco de câncer de pulmão, laringe e esôfago atribuível ao fumo. Rev Saúde Pública, 2002;36(2):129-34.

15. MICALIZZI DS, et al. A conduit to metastasis: circulating tumor cell biology. Genes Dev, 2017;31(18):1827-40.

16. MUKHERJEE, SS. The emperor of all maladies. New York: Simon \& Schuster, 2010.

17. NETO, OCLF. Metástases Hepáticas. Abordagem Atual. J Bras Med, 2014; 102(2):1-8.

18. PARKIN DM, et al. Cancer burden in the year 2000. The global picture. Eur J Cancer, 2001; 37(8):44-66.

19. PASQUALI S, et al. Systemic treatments for metastatic cutaneous melanoma. Cochrane Database Syst Ver, 2018;6;2:CD011123.

20. PINHEIRO CTS, BRITTO L. Prognóstico de Pacientes Oncológicos em uma Unidade de Tratamento Intensivo. Rev Bra Ter Int, 2001; 13(4):10-7.

21. PISANI P, BRAY F, PARKIN DM. Estimates of the world-wide prevalence of cancer for sites in the adult population. Int J Cancer, 2002; 97(1):72-81.

22. SIEGEL RL, et al. Cancer Statistics 2017, Ca Cancer J Clin 2017;67:7-30.

23. TALMADGE JE, FIDLER IJ. Cancer invasion and metastasis. In The pathobiology of human disease: a dynamic encyclopedia of disease mechanisms, San Diego: Elsevier, 2014; 423-433p.

24. TAZIKI MH, et al. Epidemiology of Head and Neck Cancers in Northern Iran: A 10-Year Trend Study From Golestan Province. Arch Iran Med, 2018;21(9):406-11. 